\title{
Flight Control of a 1-DOF Helicopter System using a Sliding Mode Controller for Disturbance Rejection
}

\author{
Cuero Jairo ${ }^{1}$, Vargas Javier ${ }^{2}$ and Jacinto Edwar ${ }^{3}$ \\ ORCIDs: 0000-0001-8416-7032 (Jairo), 0000-0001-6208-8659(Javier), 0000-0003-4038-8137(Edwar) \\ ${ }^{1}$ EYSI, MACRYPT Research Group, Universidad de los Llanos, Colombia. \\ ${ }^{2}$ EYSI, MACRYPT Research Group, Universidad de los Llanos, Colombia. \\ ${ }^{3}$ SIE Research Group, Universidad Distrital, Colombia.
}

\begin{abstract}
This document presents the design, implementation, and evaluation of a sliding mode controller to stabilize the angular position of a 1-DOF helicopter. The dynamic model of the helicopter was found using the system identification technique with the MATLAB toolbox. Also, a classic PID controller was designed to compare the behavior of both controllers and their robustness and disturbances rejection. The simulation of the control strategies was carried out in Simulink and programed on the STM32F411 microcontroller. The PID controller performed well under normal operating conditions, but, with a sustained disturbance at the input, the system output with the PID controller began to oscillate due to perturbance. The Sliding Mode Controller was not the best in the transient state. However, it did show robustness to disturbance, i.e., under the same conditions when the PID controller could not guarantee and stabilize the output, the SMC performed better, with a little slight oscillation in steady-state but without leaving the criterion of $2 \%$ of the reference value in the input.
\end{abstract}

Keywords: Nonlinear system, Sliding Mode Control, PID Controller, Helicopter.

\section{INTRODUCTION}

Industry 4.0 considers the use, appropriation, and development of unmanned vehicles as an emerging technology. In the case of aerial vehicles, the integration of drones into consumer electronics is increasingly evident due to the increase in both military and civil applications, including precision agriculture, shipping and delivery, and the entertainment industry; so, the UAV market is forecast to reach $\$ 40$ billion by 2025 [1]. The foray into new applications and the improvement of the current performance of these vehicles generate the need to solve a series of problems, not only construction but also operational, among which is the optimization and control of the propulsion system.

To control BLDC motor-based propulsion systems, usually, a simple PID controller is enough [2]. Several applications such as electric motors, pneumatic, electrical, hydraulic systems, and generally all the industries have employed this type of controller for decades, mostly due to its performance, simplicity, and robustness in the presence of uncertainties.

Another control technique widely used for nonlinear systems applications is the Sliding Mode Control (SMC). When the requirement of robustness is crucial for the development of the system, SMC fits well as it can operate in the presence of large uncertainties, disturbances, or non-linearities in the model [3]. $\mathrm{SMC}$ is a technique derived from variable structure systems (VSS) [4] that alters the dynamics of the system by applying high frequency switching control signals. However, the design needs special attention in many applications, as dealing with the phenomenon of chattering can be a daunting obstacle [5].

Several applications for UAV control have employed the SMC technique with some variations, for instance, the multi-variable integral sliding mode control[6] to track desired trajectories for the azimuth angle as well as the pitch angle in a nonlinear control-oriented model of a 2-DOF helicopter. The new control structure for a quadrotor helicopter that employs the leastsquares method to solve the overdetermined problem of the control input in the translational motion of a quadcopter[7] is another example. Here, the sliding mode controller provided robust tracking and stabilization of the quadcopter. Other papers also have addressed this last goal using an integral version of SMC[8], especially, a second-order chattering-free $\mathrm{SM}$ control is adopted to attenuate the chattering phenomenon or adding the backstepping technique[9][10] to the Sliding Mode Control.

Besides altitude [11] or speed controls, the robust control systems are also present in more complex systems, such as the trajectory tracking of a quadrotor [12][13], previously modeled using Euler-Lagrange[14], in which the SMC dynamically controls the altitude and the lateral movement. Real-time operation is mandatory for these purposes since any delay in the navigation system would produce that the flying artifact crashes or ends its flight abruptly. This real-time operation needs high-performance data acquisition systems and powerful devices like a personal computer, which guarantees sufficient sampling times to control the UAV.

Some applications need smaller air vehicles such as miniature helicopters or a quadrotor type MAV (Micro Air Vehicle), which must have resistance to disturbances in the air [15] or endure total or partial damage in the operation of the turbines[16]. This type of controllers requires some optimization techniques to perform the parameters tuning[17] to ensure that the sliding controller has disturbances rejection, to meet also the stability of the system, and the sampling time necessary for the engines that generate the propulsion react adequately. 
In addition to applying only a robust control method to handle the altitude or rotation of an aircraft, the high-order sliding mode controller (HO-SMC) can also include some optimal control techniques such as the Linear Quadratic Regulator LQR [18] to improve the performance and guarantee disturbances rejection and resistance to discontinuities in the input model of the system. This metaheuristic, called Multiobjective, allows a behavioral control of the system even with multiple degrees of freedom. Moreover, SMCs combines classical controller like PID or integral backstepping controller [19] in the natural or robust form[20] to perform observerbased designs and reduce the computational complexity. SMCs also integrate computational intelligence techniques such as fuzzy logic [21] or even more sophisticated methods that demand greater computational power like machine learning real-time operation and deep learning [22].

SMC applications are not limited to flight control. There are also other areas of engineering as the chemical industry[23], complex industrial processes, or any other mechanical-physical system that demands to be tolerant of disturbances.

This work presents the design of a Sliding Mode Controller based on the equivalent control technique to control a secondorder underdamped process. In turn, a PID controller is implemented to compare the behavior of the conventional controller versus the robust one.

\section{SYSTEM IDENTIFICATION}

The flight simulation control unit is a 1-DOF helicopter model whose propulsion is provided by a BLDC motor attached to the end of a movable shaft, which in turn is fastened to a fixed shaft and to a base that gives it stability.

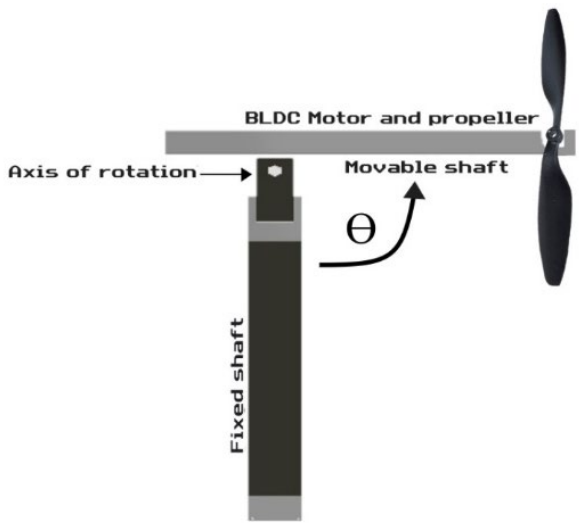

Fig. 1. Flight control unit

As the mathematical model relies on the system's behavior, then the free body diagram analysis can be employed to show the forces that act in the process and describe the dynamic. However, this requires knowing parameters such as the moment of inertia, motor constants, among others that are not easy to measure. Therefore, a black-box model is a better choice to estimate the transfer function through experimentation, which means to measure the angular displacement when a thrust force is applied.
In the transfer function, the output is the angle between the moving bar and the fixed one, and the input is the thrust force exerted by the BLDC motor at the distal end of the bar. Therefore, the relationship between the pulse width applied to the motor and the thrust force was determined. Figure 2 shows the configuration the results of the experiment.

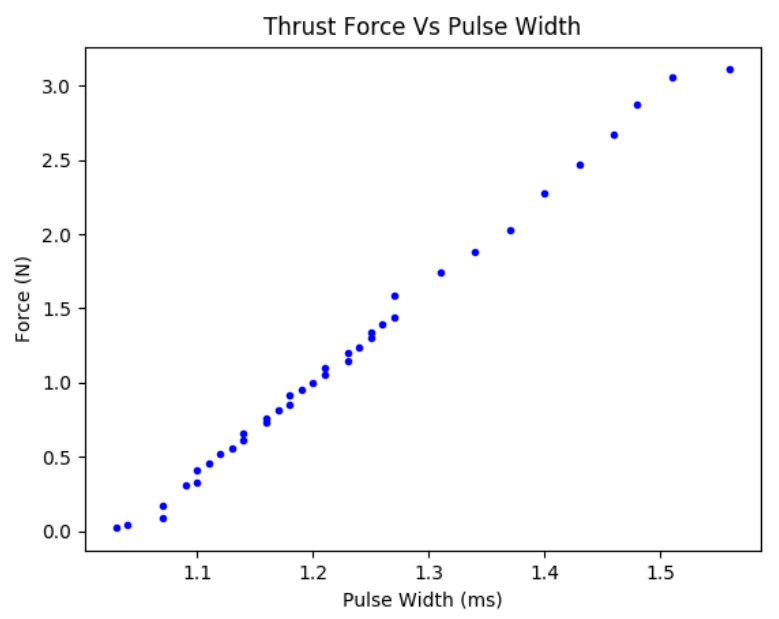

Fig. 2. Relationship between the pulse width applied to the motor and the thrust force

To solve the parameter identification problem, the pairs of input and output data are needed, so the system received a thrust force of $0.5 \mathrm{~N}$ and, it yielded the signal in figure 3 .

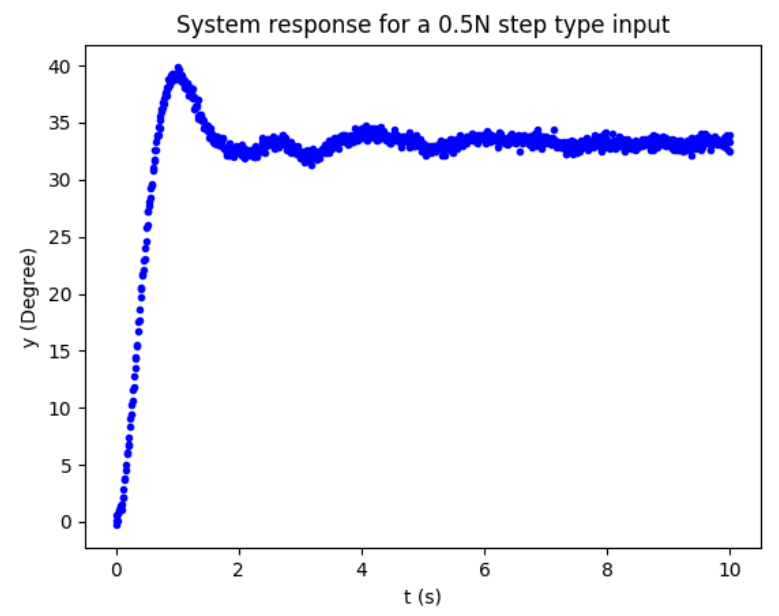

Fig. 3. Step response for a $0.5 \mathrm{~N}$ input

From the output behavior is observed that the model could be described by the characteristic equation of an underdamped system as follows:

$$
G(s)=\frac{Y(s)}{U(s)}=\frac{K}{T_{\omega}^{2} s^{2}+2 \zeta T_{\omega} s+1}
$$

Once defined the model structure, the System Identification toolbox of MATLAB helps to find the parameters of the transfer function. Equation 2 shows the result. 


$$
G(s)=\frac{Y(s)}{U(s)}=\frac{66.53}{0.07641 s^{2}+0.2721 s+1}
$$

\section{PID CONTROLLER}

The typical design of a PID controller in discrete time needs the calculation of the sampling time, this could be achieved using many techniques, here we have applied the settling time and the bandwidth criteria to set the sampling time in $10 \mathrm{~ms}$.

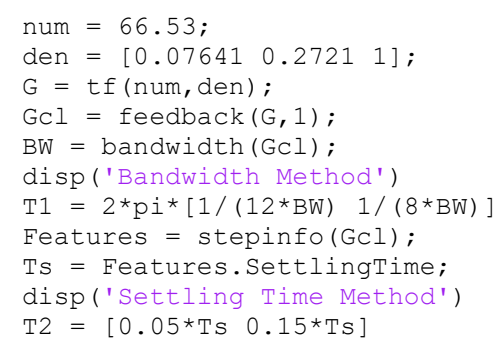

With the sampling time already defined, a classic digital PID control was designed by applying the Ziegler Nichols technique to find a suitable compensator, and then the MATLAB toolbox for the optimization of the controller parameters. The PID controller equation is in equation 3 , and table 1 shows the tunned parameters for the controller that yields the output in figure 4.

$$
C(z)=K_{p}+K_{i} \frac{T_{s}}{z-1}+K_{d} \frac{z-1}{T_{s}}
$$

Table 1. PID tunned parameters

\begin{tabular}{c|c}
\hline \multicolumn{2}{c}{ PID Parameters } \\
\hline$K_{p}$ & 0.00834 \\
$K_{i}$ & 0.0329 \\
$K_{d}$ & 0.00053 \\
\hline
\end{tabular}

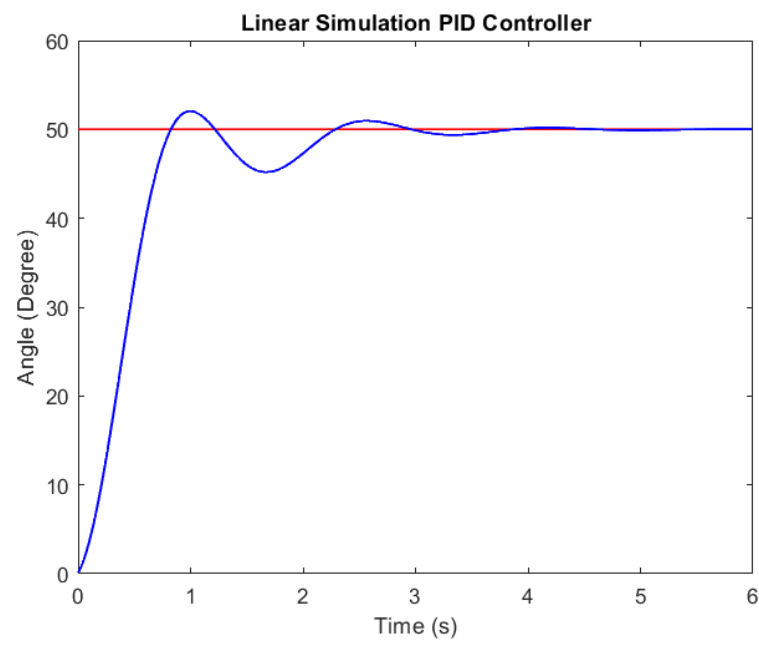

Fig. 4. system step response with the PID compensator.

\section{SLIDING MODE CONTROLLER}

The control in sliding mode requires defining a sliding surface $\mathrm{s}(\mathrm{t})$ such that $\dot{s}(t)=0$. In this case, we selected a differential integral model given by equation 4 .

$$
S(t)=\left(\frac{d}{d t}+\lambda\right)^{n} \int_{0}^{t} e(t) d t
$$

Expanding with $\mathrm{n}=2$ and grouping terms with the new parameters $\lambda_{0}=\lambda^{2}, \lambda_{1}=2 \lambda$ :

$$
S(t)=\frac{d e(t)}{d t}+\lambda_{1} e(t)+\lambda_{0} \int_{0}^{t} e(t) d t
$$

Since the error is the difference between the reference and the output, then the derivative of the reference could approximate to zero, that is:

$$
\frac{d e(t)}{d t} \cong-\frac{d y(t)}{d x}
$$

And replacing it in equation 5

$$
S(t)=-\frac{d y(t)}{d t}+\lambda_{1} e(t)+\lambda_{0} \int_{0}^{t} e(t) d t
$$

Once the sliding surface have been chosen, the control law is defined to satisfy $s(t)=0$ as a combination of a continuous and a discontinuous part as follows:

$$
u(t)=u_{C}(t)+u_{D}(t)
$$

The term $u_{c}(t)$ is also called the equivalent control and it can be obtained from system dynamics by solving the system equation for the control input and considering $\dot{s}(t)=0$.

Applying the derivative to equation 7, replacing equation 6 and equalizing it to zero.

$$
0=-\frac{d^{2} y(t)}{d t^{2}}-\lambda_{1} \frac{d y(t)}{d t}+\lambda_{0} e(t)
$$

Solving for $\mathrm{u}$ in the system equation in time domain

$$
\begin{gathered}
G(s)=\frac{Y(s)}{U(s)}=\frac{K}{T_{\omega}{ }^{2} s^{2}+2 \zeta T_{\omega} s+1} \\
\frac{1}{K}\left[\frac{T_{\omega}{ }^{2} d^{2} y(t)}{d t^{2}}+\frac{2 \zeta T_{\omega} d y(t)}{d t}+y(t)\right]=u(t)
\end{gathered}
$$

Combining equations 8 and 10 gives the equivalent control

$$
\begin{array}{r}
u_{e q}(t)=u_{c}(t)=\frac{T_{\omega}{ }^{2}}{K}\left[\left(\frac{2 \zeta}{T_{\omega}}-\lambda_{1}\right) \frac{d y(t)}{d t}+\frac{1}{T_{\omega}{ }^{2}} y(t)+\lambda_{0} e(t)\right] \\
\text { if } \lambda_{1}=\frac{2 \zeta}{T_{\omega}} \text {, then } \quad u_{c}(t)=\frac{1}{K}\left[y(t)+\lambda_{0} T_{\omega}{ }^{2} e(t)\right]
\end{array}
$$

$u_{D}(t)$ is designed based on a relay-type function like $u_{D}(t)=$ $K_{D} * \operatorname{sign}(S(t))$, which can be modified to reduce the chattering as:

$$
u_{D}(t)=K_{D} \frac{S(t)}{|S(t)|+\delta}
$$

Then, the complete control law will be the sum of equation 11 and 12 .

$$
u(t)=\frac{1}{K}\left[y(t)+\lambda_{0} T_{\omega}{ }^{2} e(t)\right]+K_{D} \frac{S(t)}{|S(t)|+\delta}
$$

The values of $\mathrm{K}$ and Tw are found by converting the equation 2 into the form of equation 1.

$$
G(s)=\frac{Y(s)}{U(s)}=\frac{66.53}{(0.2764)^{2} s^{2}+2 * 0.4922 * 0.2764 s+1}
$$


From equation $14, T_{\omega}=0.2764$ and $\zeta=0.4922$ then $\lambda_{1}=3.56$. The behavior of the sliding surface depends on the values of $\lambda_{0}$ and $\lambda_{1}$, so if a critically damped behavior is desired then $\lambda_{0}$ must be $0.25 \lambda_{1}{ }^{2}$, that is $\lambda_{0}=3.17$.

$K_{D}$ and $\delta$ are tuning parameters for the discontinuous part of the controller, a first attempt with $K_{D}=1$ and $\delta=0.1$ produced the signals in figure 5 .
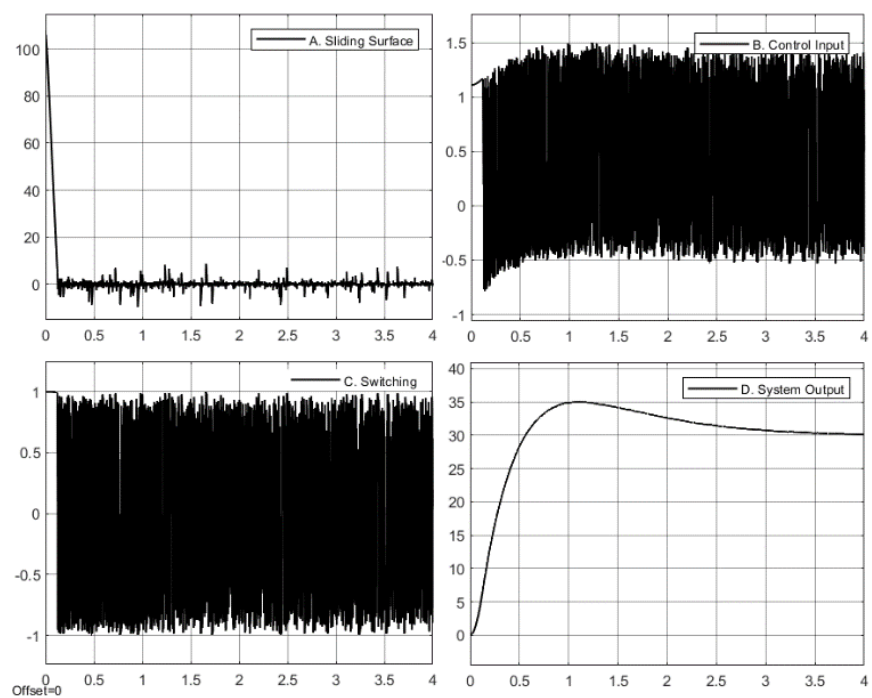

Fig. 5. Sliding surface (A), control input (B), Switching surface (C) and system output (D) for $K_{D}=1$ and $\delta=0.1$

Although the output of the system (D) seems to be adequate, a high-frequency oscillation is observed in both the control action (B) and switching surface (C), caused by the discontinuous nature of the control action. This behavior, known as the chattering phenomenon, is also present in the sliding surface (A). So, these values are unsuitable since the chattering eventually creates a problem of wear and tears in the mechanical parts and vibrations in the shaft of the helicopter.
A better response with a lower chattering is obtained using $K_{D}=0.35$ and $\delta=0.75$. Figure 6 shows the optimal system response.
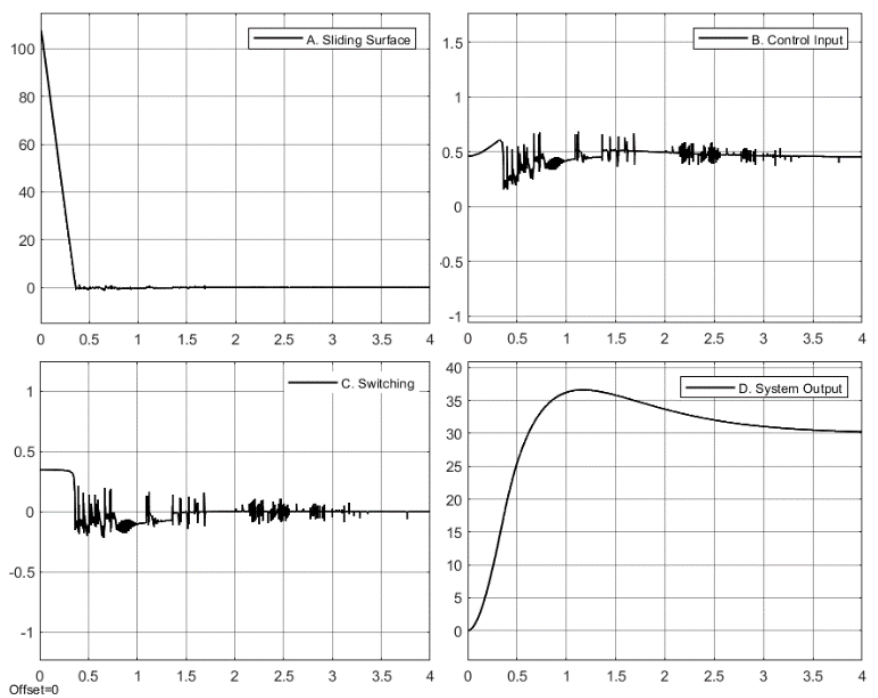

Fig. 6. Optimal response with the tuned parameters

Finally, in table 2 are the SMC values that were tuned to get an optimized behavior, and figure 7 shows the complete implementation of SM controller in Simulink

Table 2. SMC tunned parameters

\begin{tabular}{c|r}
\hline \multicolumn{2}{c}{ SMC Parameters } \\
\hline$\lambda_{0}$ & 3.17 \\
$\lambda_{1}$ & 3.56 \\
$K_{D}$ & 0.35 \\
$\delta$ & 0.75 \\
\hline
\end{tabular}

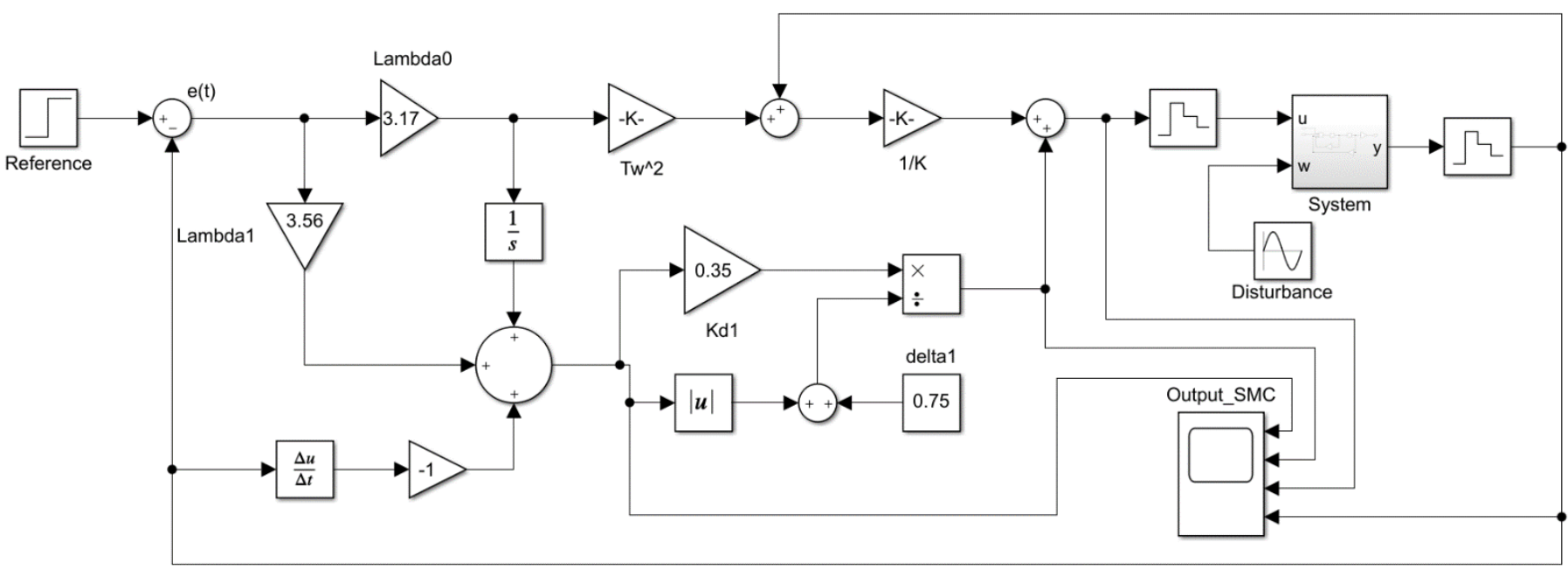

Fig. 7. Sliding Mode Controller Diagram in Simulink 


\section{PID CONTROLLER VS SM CONTROLLER}

Figure 8 plots the system response with the PID and Sliding Mode Controllers for a $30^{\circ}$ reference input. Clearly, with the PID, the system reaches faster the reference input, the response exhibits just a few oscillations and a very low overshoot and, it stabilizes in less than two seconds. Conversely, the SMC last the double of time to reach the steady-state and its overshoot is around $20 \%$.

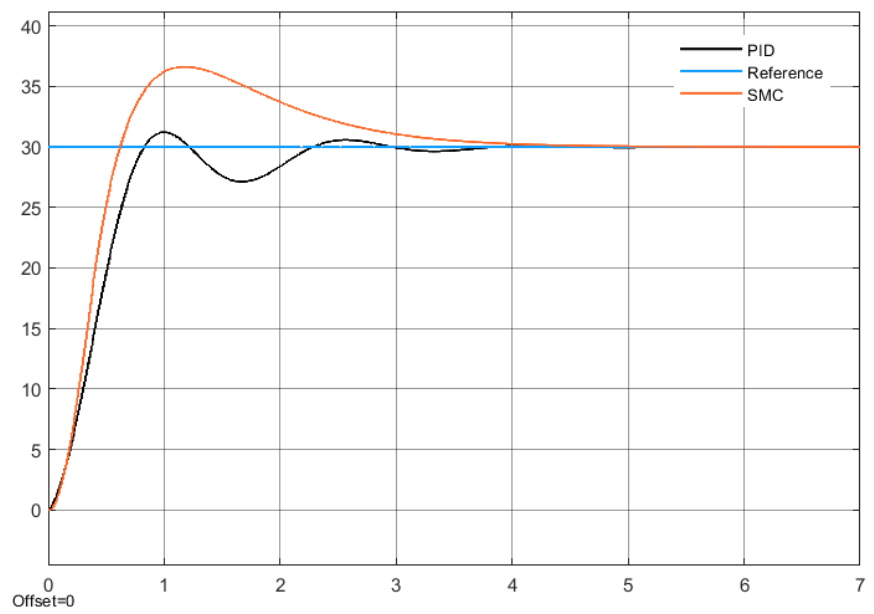

Fig. 8. Behavior of the PID and SM controllers in the absence of disturbances

The foremost advantage of a sliding mode controller is its robustness before disturbances, so, for the same $30^{\circ}$ degree reference input, a sinusoidal disturbance has been added and, figure 9 shows the system response. The SMC barely changes due to perturbation, but the PID starts oscillating around the setpoint.

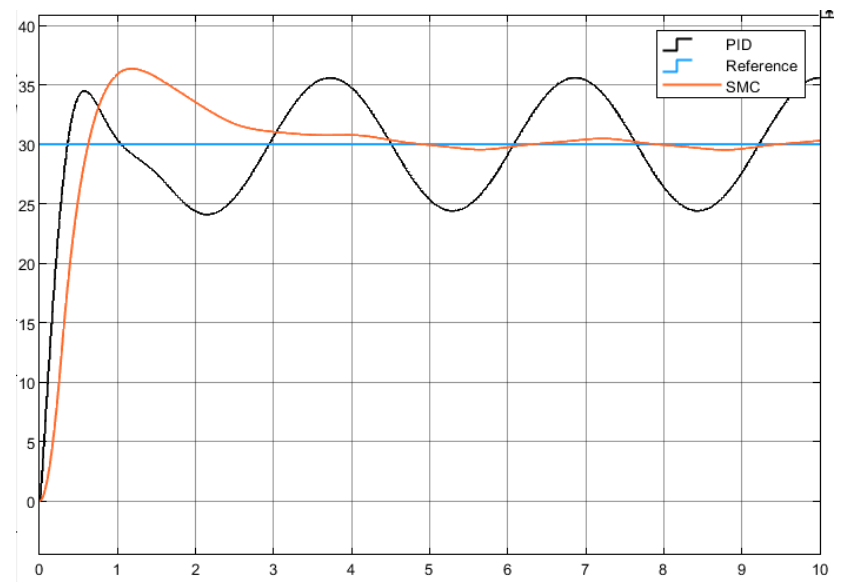

Fig. 9. Behavior of PID and SM controllers in the presence of disturbances

\section{CONCLUSIONS}

The dynamic equations that describe the behavior of the elevation axis led to the 1-DOF Helicopter model obtention. This model was linearized and experimentally validated through the system identification technique in MATLAB. Then, the PID and the Sliding Mode controllers designed around the system dynamic controlled the angular position of the elevation axis. The simulation results met the proposed design specifications, and the controllers were tested on the Simulink platform and implemented on an STM32F411 microcontroller for evaluation in the helicopter model.

The designed PID controller performed well under normal operating conditions, stabilized the system in a time of $2 \mathrm{~s}$, and with an overshoot of only $5 \%$. In contrast, the system with the controller in slide mode exhibited a significant overshoot of $20 \%$, and its stabilization time was $4 \mathrm{~s}$. However, under abnormal conditions, with a sustained disturbance at the input of the system, the PID controller showed higher sensitivity since when the perturbation started, the output of the system began to oscillate. On the other hand, the controller in sliding mode gave the system robustness since it did not react to the same disturbance applied to the input; the output presented a slight oscillation in steady-state but without leaving the criterion of $2 \%$ of the reference value in the input.

In future works, we recommend a cascade form with both controllers, so the system gets robustness to disturbances and uncertainty in the model parameters, and simultaneously presents a better behavior in the steady and transient state, that is, fast response with an overshoot less than $5 \%$.

\section{ACKNOWLEDGEMENT}

This work was supported and funded by the Directorate General Research at Universidad de Los Llanos, Colombia, and under the research project $\mathrm{N}^{\circ}$ C09-F02-005-2019.

\section{REFERENCES}

[1] W. Xu, "GIV 2025 Unfolding the Industry Blueprint of an Intelligent World," Huawei Tech., 2018, [Online]. Available: http://www.huawei.com/minisite/giv.

[2] A. L. Salih, M. Moghavvemi, H. A. F. Mohamed, and K. S. Gaeid, "Flight PID controller design for a UAV quadrotor," Sci. Res. Essays, vol. 5, no. 23, pp. 36603667, 2010.

[3] X. S. Ligang Wu, Peng Shi, Sliding mode control of uncertain parameter-switching hybrid systems. Chichester, U.K.: John Wiley \& Sons, Ltd, 2014.

[4] J. Ayala Taco, R. Gutiérrez, S. Guerra Jiménez, and A. Fernandez Correa, "Diseño e implementación de un control dual por modos deslizantes para un convertidor Buck CD-CA.," Cienc. e Ing. Neogranadina, vol. 25, no. 1, p. 91, 2015, doi: $10.18359 /$ rcin. 436 .

[5] W. Boukadida, A. Benamor, H. Messaoud, and P. Siarry, "Multi-objective design of optimal higher order sliding mode control for robust tracking of 2DoF helicopter system based on metaheuristics," Aerosp. Sci. Technol., vol. 91, pp. 442-455, 2019, doi: 10.1016/j.ast.2019.05.037. 
[6] S. S. Butt and H. Aschemann, "Multi-variable integral sliding mode control of a two degrees of freedom helicopter," IFAC-PapersOnLine, vol. 28, no. 1, pp. 802-807, 2015, doi: 10.1016/j.ifacol.2015.05.129.

[7] S. Raniprima, B. Hidayat, and N. Andini, "Digital image steganography with encryption based on rubik's cube principle," ICCEREC 2016 - Int. Conf. Control. Electron. Renew. Energy, Commun. 2016, Conf. Proc., pp. 198-201, 2017, doi: 10.1109/ICCEREC.2016.7814972.

[8] T. Jiang, D. Lin, and T. Song, "Novel integral sliding mode control for small-scale unmanned helicopters," J. Franklin Inst., vol. 356, no. 5, pp. 2668-2689, 2019, doi: 10.1016/j.jfranklin.2019.01.035.

[9] Z. Jia, J. Yu, Y. Mei, Y. Chen, Y. Shen, and X. Ai, "Integral backstepping sliding mode control for quadrotor helicopter under external uncertain disturbances," Aerosp. Sci. Technol., vol. 68, no. May, pp. 299-307, 2017, doi: 10.1016/j.ast.2017.05.022.

[10] M. E. Antonio-Toledo, E. N. Sanchez, A. Y. Alanis, J. A. Flórez, and M. A. Perez-Cisneros, "Real-Time Integral Backstepping with Sliding Mode Control for a Quadrotor UAV," IFAC-PapersOnLine, vol. 51, no. 13, pp. 549-554, 2018, doi:

10.1016/j.ifacol.2018.07.337.

[11] B. Sumantri, N. Uchiyama, and S. Sano, "Least square based sliding mode control for a quad-rotor helicopter and energy saving by chattering reduction," Mech. Syst. Signal Process., vol. 66-67, pp. 769-784, 2016, doi: 10.1016/j.ymssp.2015.05.013.

[12] O. Mofid et al., "Development of a robust attitude control for nonidentical rotor quadrotors using sliding mode control," Aerosp. Sci. Technol., vol. 15, no. 5, pp. 59-63, 2019, doi: 10.1016/j.ifacol.2018.07.337.

[13] O. Mofid et al., "Fixed-time trajectory tracking for a quadrotor with external disturbances: A flatness-based sliding mode control approach," Aerosp. Sci.

Technol., vol. 15, no. 5, pp. 59-63, 2019, doi: 10.1016/j.ifacol.2018.07.337.

[14] G. Perozzi, D. Efimov, J. M. Biannic, and L. Planckaert, "Trajectory tracking for a quadrotor under wind perturbations: sliding mode control with statedependent gains ," J. Franklin Inst., vol. 355, no. 12, pp. 4809-4838, 2018, doi: 10.1016/j.jfranklin.2018.04.042.

[15] H. Castañeda, J. Rodriguez, and J. L. Gordillo, "Continuous and smooth differentiator based on adaptive sliding mode control for a quad-rotor MAV," Asian J. Control, no. July, pp. 1-12, 2019, doi: 10.1002/asjc.2249.

[16] T. Ashitha Varghese and S. J. Mija, "Sliding Mode Control Based Design for a 6-DOF Miniature Helicopter in Hovering Flight Mode," 2019 IEEE 5th Int. Conf. Mechatronics Syst. Robot. ICMSR 2019, pp. 59-63, 2019, doi: 10.1109/ICMSR.2019.8835471.
[17] E. Baez, Y. Bravo, D. Chavez, and O. Camacho, "Tuning Parameters Optimization Approach for Dynamical Sliding Mode Controllers," IFACPapersOnLine, vol. 51, no. 13, pp. 656-661, 2018, doi: 10.1016/j.ifacol.2018.07.355.

[18] J. Xu, C. C. Lim, and P. Shi, "Sliding mode control of singularly perturbed systems and its application in quad-rotors," Int. J. Control, vol. 92, no. 6, pp. 13251334, 2019, doi: 10.1080/00207179.2017.1393102.

[19] M. Labbadi and M. Cherkaoui, "Robust adaptive backstepping fast terminal sliding mode controller for uncertain quadrotor UAV," Aerosp. Sci. Technol., vol. 93, p. 105306, 2019, doi: 10.1016/j.ast.2019.105306.

[20] W. Cai, J. She, M. Wu, and Y. Ohyama, "Disturbance suppression for quadrotor UAV using sliding-modeobserver-based equivalent-input-disturbance approach," ISA Trans., vol. 92, pp. 286-297, 2019, doi: 10.1016/j.isatra.2019.02.028.

[21] S. Zeghlache and N. Amardjia, "Real time implementation of non linear observer-based fuzzy sliding mode controller for a twin rotor multi-input multi-output system (TRMS)," Optik (Stuttg)., vol. 156, pp. 391-407, 2018, doi: 10.1016/j.ijleo.2017.11.053.

[22] X. Yao and Z. Chen, "Sliding mode control with deep learning method for rotor trajectory control of active magnetic bearing system," Trans. Inst. Meas. Control, vol. 41, no. 5, pp. 1383-1394, 2019, doi: $10.1177 / 0142331218778324$.

[23] C. T. Chen and S. T. Peng, "Design of a sliding mode control system for chemical processes," J. Process Control, vol. 15, no. 5, pp. 515-530, 2005, doi: 10.1016/j.jprocont.2004.11.001. 